\title{
Melatonin is a biomarker of circadian dysregulation and is correlated with major depression and fibromyalgia symptom severity
}

This article was published in the following Dove Medical Press journal: Journal of Pain Research

\author{
Wolnei Caumo ${ }^{1-3}$ \\ Maria Paz Hidalgo 4,5 \\ Andressa Souza ${ }^{6}$ \\ Iraci LS Torres ${ }^{1,7}$ \\ Luciana C Antunes ${ }^{8}$
}

'School of Medicine, Universidade Federal do Rio Grande do Sul (UFRGS), Porto Alegre, Brazil;

2Pain and Palliative Care Service at Hospital de Clínicas de Porto Alegre (HCPA), Laboratory of Pain and Neuromodulation at UFRGS, Porto Alegre, Brazil; ${ }^{3}$ Pain and Anesthesia in Surgery Department, School of Medicine, UFRGS, Porto Alegre, Brazil; ${ }^{4}$ Psychiatry Department, School of Medicine, UFRGS, Porto Alegre, Brazil; ${ }^{5}$ Laboratorio de Cronobiologia e Sono do Hospital de Clinicas de Porto Alegre; Porto Alegre, Brazil; ${ }^{6}$ Postgraduate Program in Health and Human Development, La Salle Universitary Center, Canoas, Brazil; ${ }^{7}$ Pharmacology Department, Instituto de Ciências Básicas da Saúde, UFRGS, Porto Alegre, Brazil; ${ }^{8}$ Centro de Ciências da Saúde - Departamento de Nutrição da Universidade Federal De Santa Catarina, Florianopolis, Brazil

Correspondence: Wolnei Caumo Laboratory of Pain and Neuromodulation, School of Medicine at UFRGS, Hospital de Clínicas de Porto Alegre, Street Ramiro Barcelos, Porto Alegre, RS, 90035-003, Brazil

Email wcaumo@hcpa.edu.br
Objective: This study compared urinary 6-sulfatoxymelatonin (aMT6s) over 24 hours among fibromyalgia (FM), major depression disorder (MDD), and healthy control (HC) groups, and examined whether rhythm is correlated with depressive symptoms. To answer this question we compared the rhythm of urinary aMT6s secretion among each group in four time series: morning (06:00-12:00 hours), afternoon (12:00-18:00 hours), evening (18:00-24:00 hours), and night (24:00-06:00 hours). In the FM subjects, we assessed if the rhythm of urinary aMT6s secretion is associated with pain severity, sleep quality, number of trigger points (NTPs), and the pain pressure threshold (PPT).

Patients and methods: We included 54 women, aged 18-60 years with diagnosis of FM $(\mathrm{n}=18)$, MDD $(\mathrm{n}=19)$, and HC $(\mathrm{n}=17)$. The 24-hour urinary aMT6s was evaluated according to four standardized periods. The assessment instruments were the Hamilton Depression Rating Scale (HDRS), Pittsburgh Sleep Quality Index, and Fibromyalgia Impact Questionnaire.

Results: A generalized estimating equation revealed no difference in the daily load of aMT6s secretion among the three groups $(P=0.49)$. However, at the daily time (06:00-18:00 hours), the load secretion of aMT6s reached $41.54 \%$ and $60.71 \%$ in the FM and MDD, respectively, as compared to $20.73 \%$ in the $\mathrm{HC}(P<0.05)$. A higher score in the HDRS was positively correlated with the amount of aMT6s secretion during daytime (06:00-18:00 hours). Also, multivariate linear regression revealed that in FM subjects, the aMT6s secretion during daytime (06:00-18:00 hours) was negatively correlated with the PPTlog (partial $\eta^{2}=0.531, P=0.001$ ). However, it was positively correlated with depressive symptoms (partial $\eta^{2}=0.317, P=0.01$ ); PQSI (partial $\eta^{2}=0.306, P=0.017$ ), and NTPs (partial $\eta^{2}=0.23, P=0.04$ ).

Conclusion: A more significant load of aMT6s secretion during daytime hours was observed in MDD and FM subjects compared to HC. These findings help to comprehend the biological basis of these disorders and show how disruption in melatonin secretion is positively correlated with clinical symptoms.

Keywords: fibromyalgia, depression, pain, melatonin, 6 sulfatoxymelatonin (aMT6s).

\section{Introduction}

Fibromyalgia (FM) is a central sensitivity syndrome that comprises chronic musculoskeletal pain, cognitive dysfunctions (ie, attentional capacity and memory), fatigue, sleep disorders, and circadian rhythm disturbances. ${ }^{1}$ Major depression disorders (MDDs) and FM share neurobiological foundations, proving that emotional and noxious processing systems are intricate and overlapping. ${ }^{2}$ Incidentally, central sensitization syndrome (CS) has been extensively addressed as a mechanism underlying the occurrence 
of FM and MDD. ${ }^{2,3}$ It comprises a decrease in the top-down inhibitory activity (dysregulation of dopamine, serotonin, norepinephrine, epinephrine, and endogenous opioids $)^{4}$ and increases bottom-up excitatory activity on pain pathways. Accordingly, a growing body of evidence has characterized $\mathrm{CS}$ as the long-term result of an aberrant stress-response system $^{5}$ that amplifies sensory input.

MDD is found in one-third to one-half of patients at the time of diagnosis of $\mathrm{FM}^{6}{ }^{6}$ and the lifetime prevalence of MDD in FM patients is 4.3 times higher than in the general population. ${ }^{7}$ Also, depressed women with FM present with more severe pain, insomnia, worse overall physical condition, and lower quality of life compared to controls. ${ }^{8}$ The relationship between insomnia as a comorbidity with depression and chronic pain conditions is organically intertwined. ${ }^{2}$ Disruption of melatonin secretion is a potentially important route for the onset and aggravation in patients with FM and depression and is involved in a complex bidirectional relationship between chronic pain and MDD. ${ }^{9}$ Thus, the comorbidities of pain and depression may affect response and engagement with treatment. Despite ongoing research, the exact etiology, diagnosis, and optimal treatment for these patients are not yet established.

Likewise, FM and MDD share several common symptoms linked to the hypothalamic-pituitary-adrenal (HPA) axis and autonomic dysregulation systems for stress regulation, and their physiological markers are commonly found in FM and MDD. ${ }^{10,11}$ Cortisol and melatonin are two hormones that regulate the 24-hour pattern of bodily functions. ${ }^{12}$ Melatonin is centrally secreted by the pineal gland and exhibits a secretory rhythm with well-defined onset and offset phases in both animals and humans ${ }^{13}$ with circulating concentrations high at night and lower during the day. It is a primary circadian pacemaker quantifiable by measuring 6 sulfatoxymelatonin (aMT6s), which is its major metabolite excreted in urine. Urinary aMT6s reflects the amount of circulating melatonin related to the period between the prior urine void and the subsequent urine sample. ${ }^{14}$ The aMT6s level is stable over time $^{15}$ and measured via well-proven and reliable techniques. It has greater reliability to measure circadian phase position over other circadian markers (ie, core body temperature and cortisol) because the melatonin concentration remains relatively uninfluenced by external factors such as stress, physical activity, and excessive carbohydrate intake. In contrast, these factors can mask the cortisol and core body temperature. ${ }^{16,17}$ Actually, melatonin acts as a chemical code of the biological clock and can be a biomarker to comprehend the impact of circadian disruption on neurophysiological, behavioral, and metabolic processes.

During the hours of darkness, some studies found a lower melatonin secretion in FM patients compared to controls, which may contribute to worse sleep at night, daytime fatigue, and higher pain perception. ${ }^{18}$ In the same way, a study found a phase shift by observing an increase in melatonin from the night to the morning in subjects with unipolar depression, while a decrease from the night to the morning was seen in the control group. ${ }^{19}$ At present, the results related to the amount of melatonin secretion during daytime hours in FM and MDD, compared to controls, are mixed. However, some studies found lower levels of serum melatonin, ${ }^{18,20}$ while other similar studies did not see these differences. ${ }^{19,21}$ Thus, urinary aMT6s is a reliable measure that can help to comprehend the relationship between the circadian disruption of melatonin secretion with MDD and FM symptoms. In the same way, it can instigate the investigation regarding the relevance of therapeutic approaches that improve the biological rhythms in clinical settings to treat MDD and FM (ie, use of exogenous melatonin).

This cross-sectional exploratory study was conducted to answer two questions: 1) to compare urinary aMT6s rhythm across 24 hours among FM, MDD, and healthy control (HC) groups and if this rhythm is correlated with depressive symptom levels. To answer this question, we compared the rhythm of urinary aMT6s secretion among groups in four time series: morning (06:00-12:00 hours), afternoon (12:00-18:00 hours), evening (18:00-24:00 hours), and night (24:00-06:00 hours). 2) In the FM group, we assessed if urinary aMT6s secretion rhythm is associated with pain severity, sleep quality, number of trigger points (NTPs), and the pain pressure threshold (PPT).

\section{Patients and methods Design, settings, and participants}

The criteria for inclusion and exclusion of the three groups (FM, MDD, and HC) are presented in Figure 1. The protocol of this cross-sectional study was reviewed and approved by the Ethics Committee Board of the Clínicas Hospital de Porto Alegre (Institutional Review Board IRB 0000921) following the Declaration of Helsinki. All individuals gave oral and written informed consent before participation.

This sample comprised outpatient adult females, aged 18-65 years who can read and write. FM subjects were recruited by directly contacting them from the institutional chronic pain clinic. Also, both FM and MDD were referrals 
A

\author{
aMT6s urinary secretion \\ across day in four time \\ series:
Morning (06:00-12:00 h), Afternoon (12:00-18:00 h) Evening (18:00-24:00 h), Night (24:00-06:00 h). Hamilton Depressive Rating Scale (HDRS)

Common characteristics: Fibromyalgia, major depression, healthy subjects (females, aged 18-65 years, do not have shift workers or have traveled trans meridionally during the month preceding the study). Recruitment: clinical outpatients at a tertiary hospital (FM and MDD) and in primary health care units Healthy controls $(\mathrm{HC})$ were recruited via newspaper publicity
Healthy controls (HC) $(n=17)$.

Recruitment: 32 patients were screened, 12 subjects did not meet the inclusion criteria and three did not consent to participate.

Inclusion criteria: Subjects without a diagnosis of acute or chronic disease or medication use.

Exclusion criteria: Any acute or chronic pain, recent use of analgesics, corticosteroids, or medications with known effects on the central nervous system. They were not included if they reported abuse of alcohol or psychotropic substances in the 6 months prior to the screening.

Major Depression Disorder (MDD) ( $\mathrm{n}=19)$

Recruitment: 49 patients were screened, 30 patients were excluded because they did not meet the inclusion criteria.

Inclusion criteria: Patients with the first episode of MDD recruited in primaty health care units or psychiatric outpatient clinic with a diagnosis confirmed by the structured clinical interviews by two independent psychiatrists.

Exclusion criteria: Used antidepressant or any psychotropic drug during the last month or used beta-blockers, pregnancy, other chronic clinical disorder, used alcohol, or illicit drug abuse.

B

C
Fibromyalgia (FM) ( $\mathrm{n}=18)$

Recruitment: 60 patients were screened and 42 were excluded ( 37 did not meet the inclusion criteria. and five did not consent to participate).

Inclusion criteria: Diagnoses criteria for FM according to the American College of Rheumatology (VAS) $\geq 50 \mathrm{~mm}$ during most of the days of the last 3 months.

Exclusion criteria: Used antidepressant or any psychotropic drug during the last month or used beta-blockers, pregnancy, other chronic clinical disorder, used alcohol, or illicit drug abuse.

\section{Assessments in fibromyalgia group}

Pain symptoms, Pittsburgh Sleep Quality Index (PSQI), number of trigger points (NTPs), pain pressme threshold (PPT).

Figure I (A) The measurement of 6-sulfatoxymelatonin (aMT6s) urinary in four time series: morning (06:00-12:00 hours), afternoon (I2:00-I8:00 hours), evening (I8:0024:00 hours), and night (24:00-06:00 hours). (B) The flow of the study with the inclusion and exclusion criteria of the three groups: depressive patients, fibromyalgia, and healthy subjects. (C) Assessments of fibromyalgia symptoms (pain symptoms, sleep quality, number of trigger points and pain threshold) to correlate with aMT6s urinary secretion across daytime.

from Basic Health Unit at Hospital de Clínicas de Porto Alegre. If subjects met the inclusion criteria based on the results of a screening questionnaire by phone, they were invited for medical evaluation, medical history collection including a detailed description of their symptoms to confirm the diagnosis by standardized criteria presented in Figure 1. A variable work schedule (ie, shift work) was an exclusion criterion as well as transmeridional travel during the month preceding the study. None of the subjects were in their menstrual period.

The diagnosis for FM and MDD groups were performed by standard assessment protocols applied by physicians with more than 10 years of experience in pain or psychiatric care, respectively. We included outpatients with their first episode of MDD who received medical care, but they did not use antidepressant or other psychotropic medications within the last month. In the same way, those included in the FM group could not have used antidepressant, anticonvulsant, or benzodiazepine medications within the last month since medicines can reduce melatonin secretion at night. The inclusion and exclusion criteria according to each group are presented in Figure 1.

Volunteers were recruited from the general population by advertisement postings in the universities and in public places in the Porto Alegre area. They were carefully screened by phone to answer a structured questionnaire to ensure that they were healthy and free of medicines and drugs, and had no history of psychiatric disorders. They were excluded if they presented scores higher than 13 on the Beck Depression Inventory. ${ }^{22,23}$

\section{Outcomes}

The primary outcome was comparing the rhythm of urinary aMT6s secretion over a 24 hours period in FM, MDD, and HC 
groups, each into four 6-hour series: morning (06:00-12:00 hours), afternoon (12:00-18:00 hours), evening (18:00-24:00 hours), and night (24:00-06:00 hours). Outcomes in FM group: the primary outcome was the PPT. Secondary outcomes included sleep quality, depressive symptoms, NTPs and the impact of FM symptoms on quality of life. The primary interest factor in the study was the rhythm of aMT6s urinary secretion over 24 hours.

\section{Instruments and assessments}

All participants completed a standardized sociodemographic questionnaire. A psychiatrist with experience in psychological assessment applied all tests, which had been validated for the Brazilian population.

\section{Structured clinical interview for DSM-IV (SCID)}

This instrument consists of a semi-structured diagnostic interview created from $D S M-I V$. The answers will identify the presence or absence of the symptoms, scored according to the judgment of the evaluator. It is composed of 10 modules, which can be used in a combined or independent way. In the study, the "A" module was used to diagnose mood episodes (bipolar and major depressive disorders). The translation and adaptation of this clinical interview into the Portuguese language present, in general, good reliability for mood disorders. ${ }^{24}$

\section{Depressive symptoms}

The Hamilton Depression Rating Scale (HDRS) based on 21 questions was used to measure depressive symptoms; the score ranges from 0 to $62 .{ }^{25}$ The HDRS item varies from 0 to 2 or $0-4$. Total score of $0-7$ is considered normal, and ratings equal to 20 or higher indicate moderate to severe depression. The adapted version for the Brazilian population showed an adequate capacity to discriminate individuals with depressive symptoms. The area under the curve (AUC) was 0.97 (95\% CI, 0.94-0.99) ${ }^{26}$ and the Cronbach's alpha reliability coefficient was 0.78 . The reliability coefficients in the Brazilian version are similar to that of the pooled mean for the alpha coefficient found in a random effects model of a meta-regression analysis 0.79 (95\% CI $0.77-0.81) .{ }^{27}$

\section{Assessments of sleep quality, disability, and PPT}

In the FM group, additional measurements were taken to assess specific symptoms. These measures included the Pittsburgh Sleep Quality Index (PSQI) to evaluate sleep quality. ${ }^{28}$ The Fibromyalgia Impact Questionnaire (FIQ) was applied to assess the set of symptoms that comprise FM. ${ }^{29}$ It consists of 10 domains, and the maximum possible score is 100. Taking into account that FM patients usually uses rescue analgesics at irregular intervals from week to week according to their pain level, for data analyses, we included the analgesic use as a dichotomous variable: the analgesic use of more than 4 days per week or the analgesic use equal to or less than 4 days per week.

To assess PPT, we asked patients to differentiate the perception of the pressure of the "onset of pain perception." They were instructed to report verbally as soon as the perception of pain began. The electronic algometer (J Tech Medical Industries, Greenwich, CT, USA) consists of a $1-\mathrm{cm}^{2}$ hard-rubber probe to apply over all of the tender points. The NTPs were determined using the PPT in the predefined areas by the American College of Rheumatology for FM criteria. ${ }^{30}$ The average of three successive readings at intervals of 3-5 minutes established the values of the PPT in $\mathrm{kgf} / \mathrm{cm}^{2}\left(\mathrm{lb} / \mathrm{cm}^{2}\right)$.

\section{Assessment of urinary aMT6s}

The collection of urine samples and clinical evaluation follow a standardized protocol. ${ }^{19,31}$ All participants were instructed to avoid any conditions that could expose them to bright light at night and between bedtime and the onset of sleep during the week they collected urine. They were instructed to certify adhesion to the research protocol regarding to their urine collection during an individual session with a physician in whom written guidance was supplied. In addition, on the day of urine collection, the physician queried regarding compliance to the protocol execution.

Participants were instructed to void their bladders upon rising in the morning, and they continued to collect all of their urine during the next 24 hours, including the complete sample produced the following day. It was not necessary to empty the bladder at exact times. However, we asked them for the date and time records when bladder emptying occurred. This measure was taken so that samples from each patient could be grouped into specific time periods. Each urine sample was collected in different vials ( $25 \mathrm{~mL}$ plastic containers), and all the samples were collected from the patients' homes on the second morning by a team researcher. They were appropriately transported and immediately delivered to the laboratory. We obtained urine aliquots from each container. The stipulated volume of each aliquot was proportional to the amount of urine produced in that specific period. Next, aliquots from each patient were pooled together according to the time of voiding in four time series as follows: morning 
(06:00-12:00 hours), afternoon (12:00-18:00 hours), evening (18:00-24:00 hours), and night (24:00-06:00 hours). Finally, aliquots of urine were transferred into plastic bottles and centrifuged (2,000 $\mathrm{g}, 5$ minutes), and the supernatant stored at $-80^{\circ} \mathrm{C}$ until assay to be performed. If any part of the sample presented incomplete micturition, we decided to exclude it. The collection procedures were the same for all groups.

The concentration of aMT6s in the pooled samples (allocated into four time points as previously described) was determined by enzyme immunoassay (Bulhmann ELISA kit; AlPCO Ltd, Windham, NH, USA) using a microtiter plate reader (450 nm, Micronal $\mathrm{B}, 380$ ), according to a previous protocol. ${ }^{19}$ The assay presented a sensitivity of $0.35 \mathrm{ng} / \mathrm{mL}$. We set the interassay variability at $7.3 \%$ for aMT6s levels above $3.5 \mathrm{ng} / \mathrm{mL}$, and the creatinine concentration was measured by the Jaffé method. This creatinine concentration was used as the reference to determine the aMT6s concentration $^{32}$ determined in nanograms per milligram of creatinine. The AUC was used to determine the total amount of aMT6s excreted by each patient in 24 hours. Also, we summed the amount of aMT6s by each specific 6 hours intervals. According to literature, aMT6s shows itself as a remarkable biomarker since its levels represents $90 \%$ of the melatonin released by the pineal gland. Thus, the urinary aMT6s/creatinine ratio is an indicator of nocturnal melatonin secretion. ${ }^{13}$

\section{Efforts to address potential sources of bias}

To reduce assessment bias, only one researcher was involved in all the assessments except the SCID-II, which in turn was applied by one psychiatrist. The evaluator that applied the clinical scales and PPT assessment is a physician with vast experience at the pain clinic outpatients' care. The algometer used ( $\mathrm{J}$ Tech Medical Industries) was calibrated and was acquired from a recognized provider in Brazil.

\section{Statistical analysis}

To assess if the data presented a normal distribution, we used the Shapiro-Wilk test. First, we identified factors that could change the aMT6s secretion load throughout the day (Table 1). A generalized estimating equations (GEE) analysis was run to compare each aMT6s at specific time points (morning [06:00-12:00 hours], afternoon [12:00-18:00 hours], evening [18:00-24:00 hours], and night [24:00-06:00 hours]) among groups (FM, MDD, and HC). We conducted the GEE with an exchangeable working correlation to account for the relationship between the aMT6s from a single participant in each time point. In the final models, interactions among the factors and time were also examined. Age was included as a covariate in the model because $\mathrm{HCs}$ are younger than FM and MDD patients, and it is biologically plausible that the decline in melatonin secretion is age-related. ${ }^{33}$ To identify the source of significant differences and to adjust for multiple comparisons, the Bonferroni's test was used.

We used the Spearman's rho coefficient to assess the relationship between the aMT6s secretion load at the particular time points: morning (06:00-12:00 hours), afternoon (12:00-18:00 hours), evening (18:00-24:00 hours), and night (24:00-06:00 hours) with symptoms associated with FM (sleep quality, depressive symptoms, NTPs, and QIF score) and with the PPT primary outcome. After confirming the corresponding assumptions, a final multivariate linear regression model was performed to adjust for multiple comparisons between the independent variable (ie, aMT6s at specific time points 06:00-18:00 hours). We selected aMT6s at the particular time point 06:00-18:00 hours because a higher level of melatonin during daytime hours indicates a phase delay of melatonin secretion. The dependent variables were pain measures (FIQ and PPT), depressive symptoms (HDRS), and sleep quality (PSQI). All analyses were adjusted by multiple comparisons using the Bonferroni's Multiple Comparison Test.

Table I Demographic characteristics of groups FM, MDD, and HC $(n=54)$

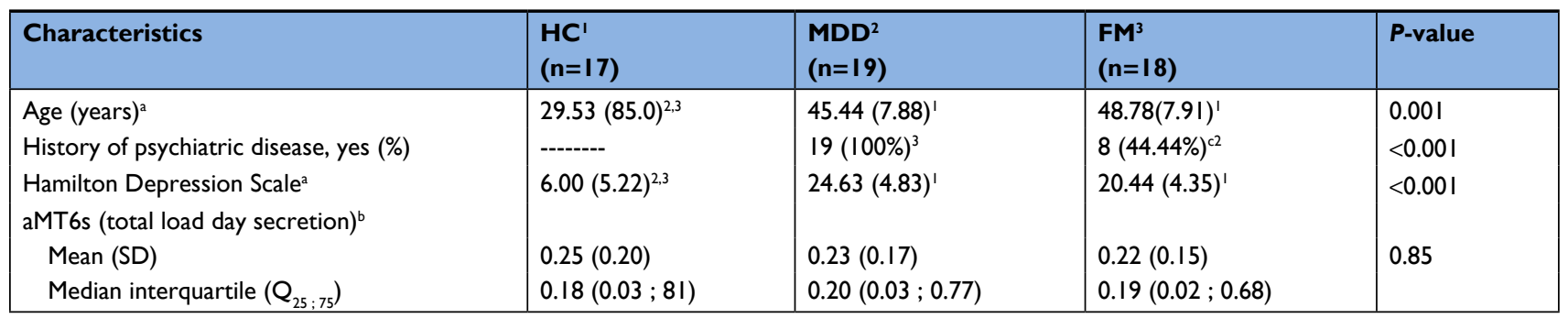

Notes: a Comparisons using ANOVA. Post hoc differences from each other at the 0.05 groups are indicated via superscript numbers. ${ }^{\mathrm{b}} \mathrm{Comparison}$ by Kruskal-Wallis test. 'Major depressive episode with dysthymia, suicidal risk, hypo-maniac episode, panic disorder, agoraphobia, social phobia, obsessive compulsive disorder, post-traumatic stress disorder, psychotic syndrome, generalized anxiety disorder, maniac-depressive disorder. Patients could have none or more than one psychiatric disorder.

Abbreviations: FM, fibromyalgia; HC, healthy control; MDD, major depression disorder. 
For the analysis regarding an association between the circadian rhythm of aMT6s secretion with FM symptoms, a prior sample size estimation indicated a study of 18 subjects. For type I and II errors of 0.05 and 0.20 , respectively, and anticipating partial eta square $\left(\eta^{2}\right)$ of 0.5 for multiple regression analysis, which allows for one predictor (in the case the aMT6s at the time point 06:00-18:00 hours), a sample size of 18 patients was estimated. ${ }^{25}$ To analyze the data we used the software SPSS version 22.0 (IBM Corporation, Armonk, NY, USA).

\section{Results}

\section{Patient characteristics: MDD, FM, and controls}

The demographic characteristics, depressive symptoms, and the daily load of aMT6s secretion are presented in Table 1. The analysis showed that compared to controls, both MDD and FM groups are older and they presented higher scores on the HDRS. However, the Kruskal-Wallis test showed that there is no difference in the total load of the aMT6s between the groups (HC, MDD, and FM).

\section{Assessment of melatonin secretion rhythm assessed by aMT6s in FM, MDD, and $\mathrm{HC}$}

Table 1 shows a statistically significant difference between FM and MDD patients compared to HC related to age and depressive symptoms. Thus, their possible confounding effect in aMT6s secretion was examined by the GEE model (Table 2). The GEE showed that there is no difference among groups in the daily load of aMT6s $\left(\chi^{2}=0.09, d f=2 ; P=0.95\right)$. In this sample, neither age nor HDRS is associated with the daily load of aMT6s secretion $\left(\chi^{2}=0.008, d f=1 ; P=0.93\right.$ and $\left.\chi^{2}=0.15, d f=1 ; P=0.69\right)$, respectively. Also, the GEE model showed that aMT6s secretion load over 24 hours across groups was not influenced significantly by age (Table 2 ).

Table 2 Generalized estimating equation model to examine the relationship between the aMT6s secretion with the group, age, and time points across the day and their interactions $(n=54)$

\begin{tabular}{|c|c|c|c|c|c|c|}
\hline Parameter & B & SE & $95 \% \mathrm{Cl}$ & $\chi^{2}$ & $d f$ & $P$-value \\
\hline Intercept & 0.63 & 0.07 & (0.49 to 0.77$)$ & 86.25 & 1 & 0.000 \\
\hline Fibromyalgia & -0.03 & 0.06 & $(-0.13$ to 0.08$)$ & 0.21 & 1 & 0.64 \\
\hline Major depression & -0.08 & 0.11 & $(-0.30$ to 0.14$)$ & 0.56 & 1 & 0.45 \\
\hline Controls & $0^{\text {referece }}$ & & & & & \\
\hline \multicolumn{7}{|l|}{ Time points } \\
\hline $06: 00-12: 00$ hours & -0.43 & 0.11 & $(-0.65$ to -0.21$)$ & 15.134 & 1 & $0.00 I^{*}$ \\
\hline $12: 00-18: 00$ hours & -0.59 & 0.08 & $(-0.75$ to -0.42$)$ & 52.123 & 1 & $0.001 *$ \\
\hline $18: 00-24: 00$ hours & -0.57 & 0.09 & $(-0.75$ to -0.39$)$ & 37.766 & 1 & $0.001 *$ \\
\hline 24:00-06:00 hours & $0^{\text {referece }}$ & & & & & \\
\hline Age & -0.004 & 0.002 & $(-0.007$ to 0.00$)$ & 3.54 & 1 & 0.06 \\
\hline Hamilton Depression Scale & 0.00 & 0.001 & $(-0.002$ to 0.003$)$ & 0.15 & I & 0.69 \\
\hline \multicolumn{7}{|l|}{ Interaction (group*age) } \\
\hline Fibromyalgia*age & -0.001 & 0.0009 & $(-0.002$ to 0.001$)$ & 0.79 & 1 & 0.37 \\
\hline Major depression*age & 0.00 & 0.0015 & $(-0.003$ to 0.003$)$ & 0.07 & 1 & 0.79 \\
\hline Controls*age & $0^{\text {referece }}$ & & & & & \\
\hline \multicolumn{7}{|c|}{ Interaction (group*time points across day) } \\
\hline Fibromyalgia*06:00-12:00 hours & -0.05 & 0.086 & $(-0.22$ to 0.11$)$ & 0.33 & 1 & 0.56 \\
\hline Fibromyalgia*12:00-18:00 hours & 0.12 & 0.069 & (0.003 to 0.19$)$ & 3.12 & 1 & $0.03 *$ \\
\hline Fibromyalgia*18:00-24:00 hours & 0.08 & 0.066 & $(-0.05$ to 0.21$)$ & 1.45 & 1 & 0.22 \\
\hline Fibromyalgia*24:00-06:00 hours & $0^{\text {referece }}$ & & & & & \\
\hline Major depression*0600-1200 hour & -0.04 & 0.089 & $(-0.22$ to 0.13$)$ & 0.27 & 1 & 0.59 \\
\hline Major depression*1200-1800 hour & 0.15 & 0.076 & (0.009 to 0.30$)$ & 4.30 & 1 & $0.03 *$ \\
\hline Major depression*1800-2400 hour & 0.15 & 0.079 & (0.007 to 0.30$)$ & 3.94 & 1 & $0.04 *$ \\
\hline Major depression $* 2400-0600$ hour & $0^{\text {referece }}$ & & & & & \\
\hline \multicolumn{7}{|c|}{ Interaction (age*time points across day) } \\
\hline Age*06:00-12:00 hours & 0.007 & 0.004 & (0.00 to 0.02$)$ & 3.670 & 1 & 0.05 \\
\hline Age*12:00-18:00 hours & 0.005 & 0.003 & (0.00 to 0.01$)$ & 4.013 & I & $0.04 *$ \\
\hline Age*18:00-24:00 hours & 0.005 & 0.002 & $(-0.001$ to 0.01$)$ & 3.064 & 1 & 0.08 \\
\hline Age*24:00-06:00 hours & $0^{\text {referece }}$ & & & & & \\
\hline
\end{tabular}

Note: ${ }^{*} P<0.05$. 
However, the analysis of the interaction between age and time points over 24 hours showed that older subjects presented a higher level of aMT6s during daytime hours between 12:00-18:00 hours (Table 2).

Also, the GEE model revealed that there is a statistically significant difference in aMT6s secretion level over 24 hours $\left(\chi^{2}=26.09, d f=3 ; P=0.001\right)$, and this difference persisted even when we examined the relationship between the time points across the day by group $\left(\chi^{2}=4.61, d f=3 ; P=0.001\right)$. The result showed an onset delay of aMT6s secretion in FM and MDD (Figure 2).

In FM subjects, the daytime pattern of aMT6s secretion (06:00 to18:00 hours) was $0.27 \mathrm{ng} \mathrm{mL}^{-1}$, and the aMT6s secretion during 24 hours was $0.65(0.92) \mathrm{ng} \mathrm{mL} \mathrm{mL}^{-1}$, that is, $41.54 \%$ of the daily secretion load occurred during the day. In the sample of patients with MDD, the daytime aMT6s secretion (06:00-18:00 hours) was $0.34(0.34) \mathrm{ng} \mathrm{mL}^{-1}$ and the aMT6s secretion during 24 hours was $0.56(0.89)$ $\mathrm{ng} \mathrm{mL} \mathrm{m}^{-1}$, that is $60.71 \%$ of the daily secretion load occurred during the day. In the HC, the daytime aMT6s secretion (06:00-18:00 hours) was $0.17 \mathrm{ng} \mathrm{mL}^{-1}$, the aMT6s secretion over 24 hours was $0.82(0.97) \mathrm{ng} \mathrm{mL} \mathrm{m}^{-1}$, and the secretion of aMT6s during the daytime was $20.73 \%$. In MDD and FM the daytime pattern of aMT6s secretion (06:00-18:00 hours) was positively correlated with HDRS (rho: 0.49 [ $P=0.03]$, 95\% CI, 0.05-0.74 and rho: 0.67 [ $P=0.001]$; 95\% CI, 0.31-086, respectively).

\section{Analysis of the association between the circadian rhythm of aMT6s secretion with FM symptoms}

The demographic and clinical characteristics of the FM group are described with more details in Table 3.

We first assessed if the aMT6s secretion over 24 hours is related to specific symptoms of FM according to four time intervals, each one of 6 hours (morning [06:00-12:00 hours], afternoon [12:00-18:00 hours], evening [18:00-24:00 hours], and night [24:00-06:00 hours]). A higher melatonin secretion at night (24:00-06:00 hours) is correlated to lower depressive symptoms (Table 4). In the same way, a higher secretion of melatonin during daytime was associated with larger NTPs and worse sleep quality. A similar finding was observed on pain threshold since a higher daytime melatonin secretion (06:00-18:00 hours) correlated negatively with lower PPT.

Variables related to pain, sleep quality, and depressive symptoms correlated with aMT6s over 24 hours in each period with 6 hour time points (morning [06:00-12:00 hours], afternoon [12:00-18:00 hours], evening [18:00-

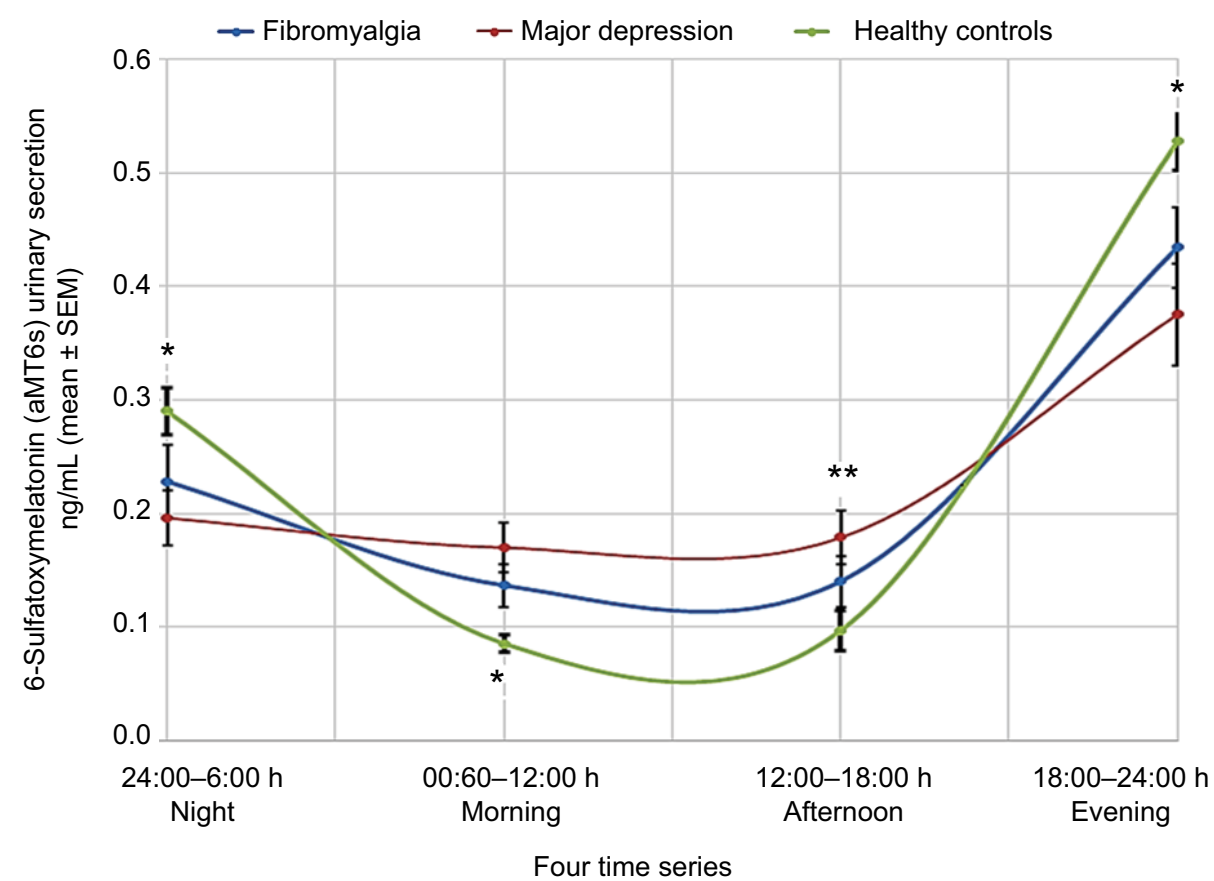

Figure 2 6-Sulfatoxymelatonin time points (aMT6s): morning (06:00-12:00 hours), afternoon (12:00-18:00 hours), evening (I8:00-24:00 hours), and night (24:00-06:00 hours) among FM, MDD, and healthy controls.

Notes: Data are presented as mean and the error bars indicate SEM. The asterisk $(*)$ indicates differences between both groups FM, MDD compared to controls. **Indicates difference of MDD compared to controls. All comparisons were performed by a GEE, followed by the Bonferroni test for post hoc multiple comparisons.

Abbreviations: FM, fibromyalgia; GEE, generalized estimating equation; HC, healthy control; MDD, major depression disorder SEM, standard error of the mean. 
Table 3 Characteristics of the sample of FM patients $(n=18)$

\begin{tabular}{|c|c|}
\hline Characteristic & $\begin{array}{l}\text { Mean } \pm \text { SD/median } \\
{\left[\text { quartile }(Q)_{25-75}\right]}\end{array}$ \\
\hline Age (years) & $48.78 \pm 7.91$ \\
\hline Body mass index $\left(\mathrm{kg} / \mathrm{m}^{2}\right)$ & $27.38 \pm 3.89$ \\
\hline Years of education (median $\left.\left(\mathrm{Q}_{25-75}\right)\right)$ & $11(6-14)$ \\
\hline Number of trigger points (median $\left(\mathrm{Q}_{25-75}\right)$ ) & $14(13-16)$ \\
\hline Employed (yes/no) & $14 / 4$ \\
\hline Smoking (yes/no) & $1 / 17$ \\
\hline Alcohol use (yes/no) & $3 / 15$ \\
\hline $\begin{array}{l}\text { Psychiatric disorder according to the } \\
\text { Structured Clinical Interview for DSM-IV } \\
\text { (yes/no)* }\end{array}$ & 08 (44.44\%) \\
\hline Major depressive episode & 6 \\
\hline Major depressive episode with dysthymia & 8 \\
\hline Dysthymia & 4 \\
\hline Suicidal risk & 3 \\
\hline Hypo-maniac episode & 2 \\
\hline Panic disorder & 5 \\
\hline Agoraphobia & I \\
\hline Social phobia & 2 \\
\hline Obsessive compulsive disorder & 4 \\
\hline Post-traumatic stress disorder & 3 \\
\hline Psychotic syndrome & 0 \\
\hline Generalized anxiety disorder & 5 \\
\hline Maniac-depressive disorder & I \\
\hline Other chronic disease (yes/no) & 8 \\
\hline Hypertension (yes/no) & 8 \\
\hline Type 2 diabetes mellitus (yes/no) & 1 \\
\hline Asthma (yes/no) & I \\
\hline $\begin{array}{l}\text { Number of analgesic doses used per week } \\
\left(\text { median }\left(Q_{25-75}\right)\right)\end{array}$ & $14(7-2 \mid)$ \\
\hline $\begin{array}{l}\text { Pain on the visual analog scale (VAS } \\
0-100 \mathrm{~mm})\end{array}$ & $70.21 \pm 14.54$ \\
\hline Pittsburgh Sleep Quality Index & $22.09 \pm 7.35$ \\
\hline Hamilton Depression Rating Scale & $24.63(4.84)$ \\
\hline Pressure Pain Threshold $\left(\mathrm{kg} / \mathrm{cm}^{2}\right)$ & $2.09 \pm 0.23$ \\
\hline Fibromyalgia Impact Questionnaire & $61.20 \pm 12.82$ \\
\hline
\end{tabular}

Note: *Patients could have none or more than one psychiatric disorder. Abbreviation: FM, fibromyalgia.
24:00 hours], and night [24:00-06:00 hours]) (Table 4) were subsequently entered as dependent variables in the multivariate linear regression model. The aMT6s (06:00-18:00 hours) during daytime hours was entered as the independent variable. The PPT logarithmic transformation was entered as the dependent variable. After adjusting for multiple comparisons using the Bonferroni test, we observed a significant negative correlation between the PPT $(\log )$, with aMT6s secretion at time interval 06:00-18:00 hours. However, the aMT6s secretion at 06:00-18:00 hours was positively associated with the HDRS, the sleep quality and the NTPs (Wilks' $\lambda=0.36, F=4.26 P=0.001$, partial $\eta^{2}=0.64$ ). The results of this adjusted multivariate model are presented in Table 5. They showed that the disruption of melatonin circadian rhythm is a factor associated with the severity of FM symptoms.

\section{Discussion}

These findings confirm our hypothesis that there is a disruption of melatonin circadian rhythm secretion in MDD and FM subjects compared to HC. Besides, they reinforce our hypothesis that a higher load of melatonin secretion during daytime hours (06:00-18:00 hours) is positively correlated with the severity of HDRS in those with FM and MDD. In FM subjects, the daytime melatonin secretion was negatively correlated with the PPT. However, a substantial aMT6s secretion during daytime hours (06:00-18:00 hours) was positively associated with the scores on the PSQI and with the NTPs. Overall, these findings indicate that a higher load of melatonin secretion during daytime hours makes subjects prone to show higher rates of depressive symptoms in FM and MDD subjects. Also, those with FM

Table 4 Correlation between the aMT6s with age, pain measures, and sleep quality in FM $(n=18)$

\begin{tabular}{|c|c|c|c|c|c|c|c|c|c|}
\hline & $\begin{array}{l}\text { aMT6s } \\
(24: 00-06: 00 \\
\text { hours) } \\
\text { Night }\left(r^{2}\right)\end{array}$ & $\begin{array}{l}\text { aMT6s } \\
(06: 00-12: 00 \\
\text { hours) } \\
\text { Morning }\left(r^{2}\right)\end{array}$ & $\begin{array}{l}\text { aMT6s } \\
(12: 00-18: 00 \\
\text { hours) } \\
\text { Afternoon }\left(r^{2}\right)\end{array}$ & $\begin{array}{l}\text { aMT6s } \\
(18: 00-24: 00 \\
\text { hours) } \\
\text { Evening }\left(r^{2}\right)\end{array}$ & $\begin{array}{l}\text { aMT6s } \\
\text { (24 hours) } \\
\left(r^{2}\right)\end{array}$ & $\begin{array}{l}\text { HDRS } \\
\left(r^{2}\right)\end{array}$ & $\begin{array}{l}\text { FIQ } \\
\left(r^{2}\right)\end{array}$ & $\begin{array}{l}\text { Number of } \\
\text { trigger } \\
\text { points }\left(r^{2}\right)\end{array}$ & $\begin{array}{l}\text { PSQI } \\
\left(r^{2}\right)\end{array}$ \\
\hline HDRS & -0.47 & 0.07 & 0.17 & $-0.50 *$ & 0.33 & & & & \\
\hline FIQ & -0.04 & 0.09 & -0.02 & 0.09 & 0.13 & 0.28 & & & \\
\hline $\begin{array}{l}\text { Number of trigger } \\
\text { points }\end{array}$ & $-0.69 * *$ & $-0.48^{*}$ & -0.05 & 0.21 & $-0.49 *$ & $0.66 * *$ & $0.58^{*}$ & & \\
\hline PSQI & 0.25 & 0.11 & $-0.58^{* *}$ & $-0.50^{*}$ & 0.34 & $0.47^{*}$ & $0.58^{*}$ & 0.08 & \\
\hline PPT & 0.27 & $-0.69 * *$ & -0.05 & 0.14 & $-0.7 I^{* *}$ & -0.39 & -0.25 & $-0.59 *$ & -0.10 \\
\hline Age & 0.24 & -0.15 & 0.02 & -0.13 & 0.05 & -0.34 & 0.28 & 0.15 & 0.09 \\
\hline
\end{tabular}

Note: $* P<0.05 ; * * P<0.01$.

Abbreviations: aMT6s, 6-sulfatoxymelatonin; HDRS, Hamilton Depression Rating Scale; PPT, Pressure Pain Threshold (kg/cm²) ; PSQI, Pittsburgh Sleep Quality Index; FIQ, Fibromyalgia Impact Questionnaire. 
Table 5 Multivariate linear regression model of the association between aMT6s (06:00-18:00 hours) during the daytime and clinical parameters of FM, including the pressure-pain threshold $(n=18)$

\begin{tabular}{|c|c|c|c|c|c|c|}
\hline \multicolumn{7}{|l|}{ Main effects } \\
\hline Dependent variables & $\begin{array}{l}\text { Type III sum } \\
\text { of squares }\end{array}$ & $d f$ & $\begin{array}{l}\text { Mean } \\
\text { square }\end{array}$ & $\boldsymbol{F}$ & $P$-value & Partial $\eta^{2}$ \\
\hline \multicolumn{7}{|l|}{ Corrected model } \\
\hline Number of trigger points & $90.927^{a}$ & I & 90.927 & 4.926 & 0.041 & 0.235 \\
\hline Hamilton Depression Rating Scale & $59.456^{\mathrm{b}}$ & I & 59.456 & 7.413 & 0.015 & 0.317 \\
\hline Pressure Pain Threshold $\left(\mathrm{kg} / \mathrm{cm}^{2}\right)$ (PPT) & $0.083^{c}$ & I & 0.083 & 16.20 & 0.001 & 0.531 \\
\hline Pittsburgh Sleep Quality Index (PSQI) & $331.076^{d}$ & I & 331.076 & 7.063 & 0.017 & 0.306 \\
\hline Fibromyalgia Impact Questionnaire (FIQ) & $\left.550.77\right|^{e}$ & 1 & 550.771 & 4.375 & 0.053 & 0.215 \\
\hline \multicolumn{7}{|c|}{ aMT6s (06:00-18:00 hours) (independent variable) } \\
\hline \multicolumn{7}{|c|}{ Beta coefficients } \\
\hline & B & SE & $\mathbf{t}$ & P-value & $95 \% \mathrm{Cl}$ & \\
\hline \multicolumn{7}{|l|}{ Dependent variable: number of trigger points } \\
\hline Intercept & 12.484 & 2.522 & 4.949 & 0.000 & \\
\hline aMT6s (06:00-18:00 hours) & 12.889 & 5.808 & 2.219 & 0.041 & \multicolumn{2}{|c|}{ (0.57 to 25.20$)$} \\
\hline \multicolumn{7}{|c|}{ Dependent variable: Hamilton Depression Rating Scale } \\
\hline Intercept & 13.965 & 1.663 & 8.400 & 0.000 & \multirow{2}{*}{\multicolumn{2}{|c|}{ (10.44 to 17.49) }} \\
\hline aMT6s (06:00-18:00 hours) & 10.423 & 3.828 & 2.723 & 0.015 & & 54) \\
\hline \multicolumn{7}{|c|}{ Dependent Variable: Pressure Pain Threshold $\left(\mathrm{kg} / \mathrm{cm}^{2}\right)$ (PPT) } \\
\hline Intercept & 2.073 & 0.108 & 19.123 & 0.000 & \multicolumn{2}{|c|}{ (1.84 to 2.30$)$} \\
\hline aMT6s (06:00-18:00 hours) & -0.552 & 0.250 & -2.214 & 0.042 & $(-1.08$ to & .03) \\
\hline \multicolumn{7}{|c|}{ Dependent Variable: Pittsburgh Sleep Quality Index (PSQI) } \\
\hline Intercept & 14.994 & 4.020 & 3.730 & 0.002 & \multicolumn{2}{|c|}{$(6.47$ to $23.5 \mathrm{I})$} \\
\hline aMT6s (06:00-18:00 hours) & 24.595 & 9.255 & 2.658 & 0.017 & \multicolumn{2}{|c|}{ (4.97 to 44.21$)$} \\
\hline \multicolumn{7}{|c|}{ Dependent Variable: Fibromyalgia Impact Questionnaire (FIQ) } \\
\hline Intercept & 49.737 & 6.587 & 7.551 & 0.000 & \multirow{2}{*}{\multicolumn{2}{|c|}{$\begin{array}{l}(35.77 \text { to } 63.70) \\
(-0.43 \text { to } 63.87)\end{array}$}} \\
\hline aMT6s (06:00-18:00 hours) & 31.723 & 15.167 & 2.092 & 0.053 & & \\
\hline
\end{tabular}

Notes: ${ }^{2} R^{2}=0.235$ (adjusted $R^{2}=0.188$ ). ${ }^{\circ} R^{2}=0.317$ (adjusted $R^{2}=0.274$ ). ${ }^{c} R^{2}=0.354$ (adjusted $R^{2}=0.317$ ). ${ }^{d}{ }^{2}=0.306$ (adjusted $R^{2}=0.263$ ). ${ }^{e}{ }^{2}=0.215$ (adjusted $R^{2}=0.166$ ). Abbreviation: FM, fibromyalgia.

are more susceptible to pain, inadequate sleep quality, and present larger NTPs.

The current findings highlighted that a more significant load of aMT6s secretion during daytime hours (06:00-18:00 hours) might be a factor that underpins the physiopathology of FM and MDD. Besides, it suggests that despite the total amount of daily aMT6s secretion not having been statistically different from the $\mathrm{HC}$, this disruption in the physiological rhythm is associated with the severity of depressive symptoms. Hence, it can help clinicians plan a more effective time schedule for the administration of melatonin to improve the symptoms related to FM and MDD. In addition, these findings may reflect a chronic stress condition, which can be a mechanism behind melatonin secretion disruption since a hyperactivation of the sympathetic system is a pathophysiological mechanism observed in both FM and MDD, as well as is part of the prolonged and sustained glucocorticoid stress-related secretion. Likewise, the alteration in rhythmic melatonin secretion likely plays a vital role developing and maintaining a dysfunctional vicious cycle of stress-related disorders. Also, the impairments of the rhythmic secretion of melatonin, in turn, affect the information transmitted to brain areas that regulate the limbic-HPA and sympatheticadrenergic-noradrenergic systems because high levels of melatonin receptors exist in the hippocampus and it is an essential regulator of both. ${ }^{34}$

The present findings corroborate data related to disruption of melatonin secretion rhythm as part of FM and MDD. Taking into account that the urinary aMT6s secretion corresponds to $90 \%$ of melatonin produced by the pineal gland, ${ }^{32}$ it is plausible that the loss of the regular day/night cycles (circadian rhythms) lead to a central role in the pathogenesis of these disorders. However, based on our findings, we cannot define if a persistent the high level of urinary aMT6s during the daytime is an expression of the sympathetic system hyperactivation as a primary compensatory mechanism or consequent to a secondary mechanism involving the pathophysiology of chronic pain and depression. If we assumed that urinary aMT6s is an indirect measure of sympathetic activity, it is possible to suppose that in FM and MDD, relentless sympathetic hyperactivity exists throughout 24 hours. Hence, it makes one more prone to desynchronize the 
melatonin secretion to environmental timing cues, and this is associated with clinical symptoms commonly observed in MDD and FM, such as worse sleep quality. Although this seems an apparent paradox (sympathetic hyperactivity with hypo-reactivity), this is a physiological response in agreement with the fundamental physiological principle that chronic hyperstimulation of $\beta$-adrenergic receptors leads to receptor desensitization and downregulation. ${ }^{34}$ Thereby, the present findings can help to improve the comprehension of the mechanism involved in the cascade of the events secondary to the disruption of sleep/wake cycle, as well as to link them to clinical symptoms observed in chronic pain and depression.

Another possible mechanism to explain the higher level of aMT6s during daytime hours (time point 06:00-18:00 hours) when physiologically we would be expected to find lower levels of aMT6s is the synthesis of melatonin in multiple extra-pineal sites. Even though we do not have consistent data to sustain this hypothesis and, until now, the literature is scarce and the described physiological mechanisms are elusive, this is a possible explanation to justify the more massive load of the daytime melatonin secretion observed in FM and MDD. This argument finds plausibility of the literature data indicating that melatonin concentrations in the extra-pineal sites exceed those in the blood (ie, brain, retina, airway epithelium, gastrointestinal tract, liver, kidney, thyroid, pancreas, immune system cells, etc). ${ }^{35}$

Although the extra-pineal melatonin secretion is a complicated process and not wholly understood, inputs of neural, endocrine, and paracrine sources participate in the regulation of melatonin synthesis and release. In this sense, it is plausible that the extra-pineal melatonin synthesis may reflect an adaptive mechanism to maintain a homeostatic equilibrium among all organs and tissues. However, we need to realize that we found a high level of urinary aMT6s during daytime. However, this finding does not allow determining the origin of this melatonin's metabolite. Indeed, it is possible to affirm that in FM and MDD, a displacement of the secretory process of melatonin in the course of 24 hours is observed, which indicates a desynchronization of melatonin secretion with the light-dark cycle.

The present findings showed that in FM and MDD there is a higher melatonin secretion during daytime hours (06:00-18:00 hours) that was associated with a higher level of depressive symptoms. Among FM patients, the more substantial number of trigger points correlates with worse sleep quality and lower PPT (Table 4). The relationship between the disruption in melatonin secretion and the propensity to mood disorders could be determined by direct neural input from the clock or indirect effects that may alter the neuroplasticity, neurotransmission, and clock gene expression of brain regions involved in emotion and mood regulation. ${ }^{36}$ Our results relating to the sleep quality in FM are aligned with previous studies on chronic pain, which found a prevalence of the sleep disorders ranging from $50 \%$ to $70 \% .{ }^{37-40}$ Even though the sleep disturbances can differ according to the pain syndrome, a previous study showed that $48.6 \%$ of people with chronic pain reported insomnia compared to $17.2 \%$ in those without chronic pain. ${ }^{41}$ Also, previous studies found increased arousal and sleep fragmentation in FM compared with controls. ${ }^{42}$ However, a bidirectional relationship exists between pain and sleep, since daytime pain is associated with reduced subsequent nighttime sleep, and poor sleep is, in turn, linked with augmented next-day pain. ${ }^{43}$ These dysfunctional processes can amplify pain and the nervous system gaining the ability to magnify pain and losing the ability to inhibit pain.

Although our results open a way to understand the relationship between the disruptions in melatonin secretion and clinical syndromes, we need parsimony in their interpretation because the design of the current study prevents determining if the dysfunction causing the disruption of melatonin rhythm secretion is the central mechanism or if it is the hyperactivation of extra-pineal melatonin secretion or both. Although it is not feasible in clinical research to determine in-loco melatonin secretion and that the urinary aMT6s is an indirect measure, it may hold critical clinical implications such as 1) to support comprehension of the bidirectional pathways between the disruption of melatonin rhythm secretion and FM and MDD; 2) to plan further studies to assess the best therapeutic approach to adjust the rhythm of melatonin secretion as a target to improve the sleep quality, which has been unfavorably, associated with pain and depression outcomes, such as a reduced quality of life; and 3) to define strategies to manage patients with a higher risk of disruption of the wake-sleep cycle as a likely factor to improve symptoms associated with FM and MDD. ${ }^{44}$ Also, it improves the comprehension of the therapeutic use of melatonin concerning pain, which has been demonstrated in clinical trials in several chronic pain conditions. ${ }^{45}$

This study is an exploratory study, and it had some limitations: First, we included only females, take into account that gender differences in pain perception and modulation are controversial. Second, psychiatric disorders are a potential confounding factor in chronic pain syndromes, and even though we used multivariate analysis, our sample size is small. In fact, we found a prevalence of $44.44 \%$ 
(8/18) of psychiatric diagnosis in the FM group (ie, anxiety, depression, and another mental disease) (Table 1). This high prevalence can be explained because the study was performed on tertiary care consulting patients. However, it is in agreement with the range of depressive and anxiety disorders found in previous studies with FM, which ranges from $20 \%$ to $80 \%$ and $13 \%$ to $63.8 \%$, respectively. ${ }^{7}$ Also, we need to realize that the emotional disturbance is intrinsic to the FM. Third, a strength of this study is that our findings converge and support the hypothesis that circadian and sleep disturbances may play a critical role in the pathophysiology of mood disorders and FM. In addition, they permit to elucidate the underpinnings of the relationships between circadian rhythm disturbances, including sleep disturbances, chronic pain, and clinical mood disorders. Fourth, although we have not found the difference among groups in a load of aMT6s secretion during 24 hours, the analyses was adjusted for the age effect since HCs were younger compared to FM and MDD. Hence, it is unlikely that controls' age would modify the present findings. Fifth, even though this method does not allow exact calculation of the levels of melatonin at a specific time of the day, a higher melatonin secretion at daytime in FM and MDD may be an intrinsic mechanism of the circadian disruption on the physiopathology of FM and MDD. Finally, we need to consider that the measurement of aMT6s in urine is a practical way to measure melatonin concentration in large psychiatric populations or patients with chronic pain in a naturalistic environment.

These results suggest that the measurement of aMT6s in urine might be a reliable biomarker of the disruption in melatonin secretion rhythm in MDD and FM compared to HC. They showed that a daytime melatonin secretion is positively correlated with the severity of depressive symptoms and with FM symptoms. Besides, they help to comprehend the biological basis of these disorders, and they suggest that this simple test might identify the level of dysfunction of melatonin secretion and be useful to contribute to individualize the clinical decision-making on the selection of the best therapeutic approaches.

\section{Acknowledgments}

Sources of support that require acknowledgment: Committee for the Development of Higher Education Personnel - CAPES - PNPD/CAPES, National Council for Scientific and Technological Development - CNPq, Postgraduate Program in Medical Sciences at the School of Medicine of the Federal University of Rio Grande do Sul, Postgraduate Research Group at the Hospital de Clínicas de Porto Alegre (FIPE-HCPA) and Brazilian Innovation Agency (FINEP). This study was supported by the following Brazilian agencies: Committee for the Development of Higher Education Personnel - CAPES - material support. PGI 011/29. Postgraduate Research Group at the Hospital de Clínicas de Porto Alegre - FIPE HCPA (material support). Number: 0000921 Brazilian Innovation Agency (FINEP) process number - 1245/13 (Torres and Caumo). Research grant: National Council for Scientific and Technological Development-CNPq (Torres 302345/2011-6 and Caumo WC-301256/2013-6).

\section{Disclosure}

The authors report no conflicts of interest in this work.

\section{References}

1. Wolfe F, Clauw DJ, Fitzcharles MA, et al. The American College of Rheumatology preliminary diagnostic criteria for fibromyalgia and measurement of symptom severity. Arthritis Care Res. 2010;62(5):600-610.

2. Maletic V, Robinson M, Oakes T, Iyengar S, Ball SG, Russell J. Neurobiology of depression: an integrated view of key findings. Int J Clin Pract. 2007;61(12):2030-2040.

3. Woolf CJ. Central Sensitization: Implications for the diagnosis and treatment of pain. Pain. 2012;152.

4. Wallace DJ, Gotto J. Hypothesis: bipolar illness with complaints of chronic musculoskeletal pain is a form of pseudofibromyalgia. Semin Arthritis Rheum. 2008;37(4):256-259.

5. Lyon P, Cohen M, Quintner J. An evolutionary stress-response hypothesis for chronic widespread pain (fibromyalgia syndrome. Pain Med. 2011;12(8):1167-1178.

6. Weir PT, Harlan GA, Nkoy FL, et al. The incidence of fibromyalgia and its associated comorbidities: a population-based retrospective cohort study based on International Classification of Diseases, 9th Revision codes. J Clin Rheumatol. 2006;12(3):124-8.

7. Fietta P, Fietta P, Manganelli P. Fibromyalgia and psychiatric disorders. Acta Biomed. 2007;78(2):88-95.

8. Segura-Jiménez V, Álvarez-Gallardo IC, Carbonell-Baeza A, et al. Fibromyalgia has a larger impact on physical health than on psychological health, yet both are markedly affected: the al-Ándalus project. Semin Arthritis Rheum. 2015;44(5):563-570.

9. Cho S, Lam C, Wesch J. Information recall bias in chronic pain and depression. J. Pain. 7, Supplement, Page S91 (2006).

10. Becker S, Schweinhardt P. Dysfunctional neurotransmitter systems in fibromyalgia, their role in central stress circuitry and pharmacological actions on these systems. Pain Res Treat. 2012;2012:1-10.

11. Ngampramuan S, Tungtong P, Mukda S, Jariyavilas A, Sakulisariyaporn C. Evaluation of autonomic nervous system, saliva cortisol levels, and cognitive function in major depressive disorder patients. Depress Res Treat. 2018;2018:1-8.

12. Chan S, Debono M. Replication of cortisol circadian rhythm: new advances in hydrocortisone replacement therapy. Ther Adv Endocrinol Metab. 2010;1(3):129-138.

13. Bojkowski CJ, Arendt J, Shih MC, Markey SP. Melatonin secretion in humans assessed by measuring its metabolite, 6-sulfatoxymelatonin. Clin Chem. 1987;33(8):1343-1348.

14. Mirick DK, Davis S. Melatonin as a biomarker of circadian dysregulation. Cancer Epidemiol Biomark Prevent. 2008;17(12):3306-3313. 
15. Pandi-Perumal SR, Smits M, Spence W, et al. Dim light melatonin onset (DLMO): a tool for the analysis of circadian phase in human sleep and chronobiological disorders. Prog Neuropsychopharmacol Biol Psychiatry. 2007;31(1):1-11.

16. Kräuchi K, Cajochen C, Werth E, Wirz-Justice A. Alteration of internal circadian phase relationships after morning versus evening carbohydrate-rich meals in humans. J Biol Rhythms. 2002;17(4):364-376.

17. Lewy AJ, Cutler NL, Sack RL. The endogenous melatonin profile as a marker for circadian phase position. J Biol Rhythms. 1999;14(3):227-236.

18. Wikner J, Hirsch U, Wetterberg L, Röjdmark S. Fibromyalgia - a syndrome associated with decreased nocturnal melatonin secretion. Clin Endocrinol. 1998;49(2):179-183.

19. Carvalho LA, Gorenstein C, Moreno RA, Markus RP. Melatonin levels in drug-free patients with major depression from the southern hemisphere. Psychoneuroendocrinology. 2006;31(6):761-768.

20. Thompson C, Franey C, Arendt J, Checkley SA. A comparison of melatonin secretion in depressed patients and normal subjects. $\mathrm{Br} J$ Psychiatry. 1988;152:260-265.

21. Rubin RT, Heist EK, Mcgeoy SS, Hanada K, Lesser IM. Neuroendocrine aspects of primary endogenous depression. XI. Serum melatonin measures in patients and matched control subjects. Arch Gen Psychiatry. 1992;49(7):558-567.

22. Warmenhoven F, van Rijswijk E, Engels Y, et al. The Beck Depression Inventory (BDI-II) and a single screening question as screening tools for depressive disorder in Dutch advanced cancer patients. Supportive Care in Cancer. 2012;20(2):319-324.

23. Gomes-Oliveira MH, Gorenstein C, Lotufo Neto F, Andrade LH, Wang YP. Validation of the Brazilian Portuguese version of the Beck Depression Inventory-II in a community sample. Rev Bras Psiquiatr. 2012;34:389-394.

24. Del-Ben CM. Reliability of the structured clinical interview for DSMIV- clinical version translated into Portuguese. Rev Bras Psiquiatr. 2001; 23:156-59. Portuguese.

25. Hamilton M. A rating scale for depression. J Neurol Neurosurg Psychiatry. 1960;23(1):56-62.

26. Freire M. Á. Hamilton Scale: study of the psychometric characteristics in a sample from Southern Brazil. J Bras Psiquiatr. 2014;63.

27. Lakens D. Calculating and reporting effect sizes to facilitate cumulative science: a practical primer for t-tests and ANOVAs. Front Psychol. 2013;4:863.

28. Buysse DJ, Reynolds CF, Monk TH, Berman SR, Kupfer DJ. The Pittsburgh sleep quality index: A new instrument for psychiatric practice and research. Psychiatry Res. 1989;28(2):193-213.

29. Marques AP, Santos AMB, Assumpção A, Matsutani LA, Lage LV, Pereira CAB. Validação da versão brasileira do Fibromyalgia Impact Questionnaire (FIQ). Rev Bras Reumatol Engl Ed. 2006;46(1):24-31.
30. Wolfe F, Smythe HA, Yunus MB, et al. The American College of Rheumatology 1990 Criteria for the Classification of Fibromyalgia. Arthritis Rheum. 1990;33(2):160-172.

31. Carvalho LA, Gorenstein C, Moreno R, Pariante C, Markus RP. Effect of antidepressants on melatonin metabolite in depressed patients. J Psychopharmacol. 2009;23(3):315-321.

32. Klante G, Brinschwitz T, Secci K, Wollnik F, Steinlechner S. Creatinine is an appropriate reference for urinary sulphatoxymelatonin of laboratory animals and humans. J Pineal Res. 1997;23(4):191-197.

33. Wurtman RJ. Age-related decreases in melatonin secretion--clinical consequences. J Clin Endocrinol Metab. 2000;85(6):2135-6.

34. Musshoff U, Riewenherm D, Berger E, Fauteck JD, Speckmann EJ. Melatonin receptors in rat hippocampus: molecular and functional investigations. Hippocampus. 2002;12(2):165-173.

35. Acuña-Castroviejo D, Escames G, Venegas C, et al. Extrapineal melatonin: sources, regulation, and potential functions. Cell Mol Life Sci. 2014;71(16):2997-3025.

36. Bedrosian TA, Nelson RJ. Timing of light exposure affects mood and brain circuits. Transl Psychiatry. 2017;7(1):e1017.

37. Atkinson JH, Ancoli-Israel S, Slater MA, Garfin SR, Dillin JC. Subjective sleep disturbance in chronic back pain. Clin. J. Pain. 1988;4(4):225-232.

38. Morin CM, Gibson D, Wade J. Self-reported sleep and mood disturbance in chronic pain patients. Clin J Pain. 1998;14(4):311-314.

39. Pilowsky I, Crettenden I, Townley M. Sleep disturbance in pain clinic patients. Pain. 1985;23(1):27-33.

40. Smith MT, Haythornthwaite JA. How do sleep disturbance and chronic pain inter-relate? Insights from the longitudinal and cognitive-behavioral clinical trials literature. Sleep Med Rev. 2004;8(2):119-132.

41. Taylor DJ, Mallory LJ, Lichstein KL, Durrence HH, Riedel BW, Bush AJ. Comorbidity of chronic insomnia with medical problems. Sleep. 2007;30(2):213-218.

42. Jennum P, Drewes AM, Andreasen A, Nielsen KD. Sleep and other symptoms in primary fibromyalgia and in healthy controls. J Rheumatol. 1993;20(10):1756-1759.

43. Raymond I, Nielsen TA, Lavigne G, Manzini C, Choinière M. Quality of sleep and its daily relationship to pain intensity in hospitalized adult burn patients. Pain. 2001;92(3):381-388.

44. Pavlova M. Circadian rhythm sleep-wake disorders. Continuum. 2017;23(4):1051-1063.

45. de Zanette SA, Vercelino R, Laste G, et al. Melatonin analgesia is associated with improvement of the descending endogenous pain-modulating system in fibromyalgia: a phase II, randomized, double-dummy, controlled trial. BMC Pharmacol Toxicol. 2014;15(1):40.
Journal of Pain Research

\section{Publish your work in this journal}

The Journal of Pain Research is an international, peer reviewed, open access, online journal that welcomes laboratory and clinical findings in the fields of pain research and the prevention and management of pain. Original research, reviews, symposium reports, hypothesis formation and commentaries are all considered for publication.

\section{Dovepress}

The manuscript management system is completely online and includes a very quick and fair peer-review system, which is all easy to use. Visit http://www.dovepress.com/testimonials.php to read real quotes from published authors. 